\title{
Stellar forensics - I. Cooling curves
}

\author{
Brad M. S. Hansen ${ }^{1,2 \star}$ and E. Sterl Phinney ${ }^{2}$ \\ ${ }^{1}$ Canadian Institute for Theoretical Astrophysics, University of Toronto, Toronto, ON M5S 3H8, Canada \\ ${ }^{2}$ Theoretical Astrophysics, 130-33, California Institute of Technology, Pasadena, CA 91125, USA
}

Accepted 1997 September 1. Received 1997 June 3; in original form 1996 November 25

\begin{abstract}
A B S T R A C T
The presence of low-mass, degenerate secondaries in millisecond pulsar binaries offers the opportunity to determine an age for the binary system independently of the rotational properties of the pulsar. To this end, we present here a detailed calculation of the evolution of a grid of low-mass $\left(<0.5 \mathrm{M}_{\odot}\right)$ helium core white dwarfs. We investigate the effects of different hydrogen layer masses and provide results for well-known optical bandpasses. We supplement the OPAL opacity calculations with our own calculations for low effective temperatures $\left(T_{\text {eff }}<6000 \mathrm{~K}\right)$ and also provide fitting formulae for the gravity as a function of mass and effective temperature. In Paper II we apply these results to individual cases.
\end{abstract}

Key words: binaries: general - stars: evolution - stars: fundamental parameters - white dwarfs.

\section{INTRODUCTION}

Studies of binary pulsars (see Phinney \& Kulkarni 1994 for references) and close double degenerate systems (Marsh, Dhillon $\&$ Duck 1995) have discovered a number of low-mass objects. The masses of these optically faint stars are estimated to be $\sim 0.1-0.5$ $\mathrm{M}_{\odot}$. Thus it is believed that these are helium core white dwarfs, which are not massive enough to ignite core helium burning and burn to carbon (Sweigart \& Gross 1978; Mazzitelli 1989). Such stars arise in binaries because the progenitor is disrupted by Roche lobe overflow at some point in its natural evolution, losing its envelope and leaving behind a low-mass, degenerate helium star (Kippenhahn, Kohl \& Weigart 1967).

Many millisecond pulsar binaries contain such dwarfs and, as such, their cooling ages offer an estimate of the age of the system, independent of the pulsar spin-down age, and thus an interesting check of the traditional assumptions made about the ages of millisecond pulsars.

Our aim in this paper is to present an extensive grid of cooling models covering the parameter space of low-mass helium core white dwarfs, much in the spirit of previous studies of the more ubiquitous carbon/oxygen core white dwarfs (Wood 1992; D'Antona \& Mazzitelli 1990 and references therein). In Paper II we apply these models to the observations of the low-mass binary pulsar population.

In Section 3 we briefly review the physical mechanisms that contribute to the white dwarf cooling process and discuss their relative importance. Section 3 describes our calculations of the low-temperature opacities necessary to obtain accurate cooling sequences for the oldest systems. In Section 4 we describe our numerical model and the tests of the code against other models from

^E-mail: hansen@cita.utoronto.ca the literature. Finally, in Section 5 we present our cooling sequences and describe the details of the cooling models. We provide a brief summary of our numerical procedures in an appendix.

\section{WHITE DWARF COOLING}

The basic qualitative picture of white dwarf cooling is a well-known one, going back to Mestel (1952). The star is supported by the pressure of a degenerate electron gas while the heat content is dominated by the thermal reservoir of non-degenerate ions, which can form a gas or a crystalline solid, depending on the Coulomb coupling parameter $\Gamma=(Z e)^{2} / a k T$, where $a$ is the radius of the Wigner-Seitz sphere surrounding each ion. While the luminosity is dominated by the loss of thermal energy, young, hot white dwarfs may also have a contribution from residual hydrogen burning at the base of the hydrogen envelope.

The energy transport throughout most of the star is dominated by conduction due to the degenerate electrons, which keeps the core almost isothermal. The core is surrounded by a thin, non-degenerate envelope where the energy transport is by radiative diffusion and, at late times, by convection (Böhm et al. 1977). This is the region of least efficient energy transport and thus it determines the rate at which the star cools. Hence the chemical composition of these outer layers is important. The high gravities of white dwarfs lead to gravitational settling (Schatzmann 1958; Dupuis et al. 1992 and references therein) which results in chemical separation in the white dwarf envelope, and hence the atmospheric opacity is dominated by the lightest species remaining on the surface, usually either hydrogen or helium.

Since the cooling is determined by the radiative transport, the accuracy of the cooling curves will be dependent on the accuracy of the opacities that we use. Over most of the cooling sequence we use the radiative opacities from the OPAL group (Rogers \& Iglesias 1992), which are good down to $T=6000 \mathrm{~K}$. For conductive 
opacities we use the results of Itoh et al. (1983) and Mitake, Ichimaru \& Itoh (1984) for the region where the ions are gas or liquid and the electrons degenerate (helium cores do not reach crystallization temperatures within a Hubble time). At temperatures and densities not covered by the Itoh results, we use the older conductivities of Hubbard \& Lampe (1969). This still leaves the region $T<6000 \mathrm{~K}$ unaccounted for. It is important because many of the objects that we will discuss in Paper II have $T_{\text {eff }} \sim 4000 \mathrm{~K}$. To address this issue, we have calculated $Z=0$ opacities for an arbitrary mix of hydrogen and helium using primarily the input physics of Lenzuni, Chernoff \& Salpeter (1991, henceforth LCS). In particular, we calculate the opacities from the collisionally induced absorption (CIA) by molecular hydrogen. We describe this briefly below.

\section{LOW-TEMPERATURE $Z=$ O OPACITIES}

We calculate the opacity of a gas containing $\mathrm{H}, \mathrm{He}, \mathrm{H}_{2}, \mathrm{H}^{-}, \mathrm{He}^{-}$, $\mathrm{H}^{+}, \mathrm{H}_{2}^{+}, \mathrm{H}_{3}^{+}, \mathrm{He}^{+}$and electrons. We assume ideal gas and local thermodynamic equilibrium (LTE). From the equation of state of Saumon, Chabrier \& Van Horn (1995), we determine that the assumption of an ideal gas is good for $\rho<10^{-2} \mathrm{~g} \mathrm{~cm}^{-3}$. The partition functions and cross-sections are taken largely from LCS. For the calculation of collisionally induced molecular opacities we use the fits of LCS for the $\mathrm{H}_{2}-\mathrm{H}_{2}$ roto-vibrational transitions (with corrections for typographical errors, for which we thank Dr Chernoff), after checking it with the original code (Borysow \& Frommhold 1990), kindly supplied to us by Dr A. Borysow. The roto-translational contribution is recalculated using another, more recent, code (Zhang \& Borysow 1995) again supplied by Dr Borysow. We also recalculate the $\mathrm{H}_{2}-\mathrm{He}$ opacity following LCS, although most of our atmospheres end up with pure $\mathrm{H}$ or $\mathrm{He}$ compositions.

When we use the composition $X=0.72$ and $Y=0.28$, we find good agreement with LCS except at the high-temperature, highdensity end $\left(T \sim 6000-7000 \mathrm{~K}\right.$ and $\left.\rho \sim 10^{-3} \mathrm{~g} \mathrm{~cm}^{-3}\right)$, where we have a 10-20 per cent error. We attribute this to our LTE treatment of the $\mathrm{H}^{-}$ion. LCS note that the equilibrium abundance of this ion is affected by the radiation field at almost any temperature because of its low dissociation energy. Since the OPAL opacities reach down to $6000 \mathrm{~K}$ and our atmospheres are convective in this region anyway (see Fig. 6, later), we opt for simplicity and use our LTE results.

For the case of a pure hydrogen atmosphere of moderate density $\rho \sim 10^{-5} \mathrm{~g} \mathrm{~cm}^{-3}$, the opacity is dominated above $\sim 3000 \mathrm{~K}$ by the

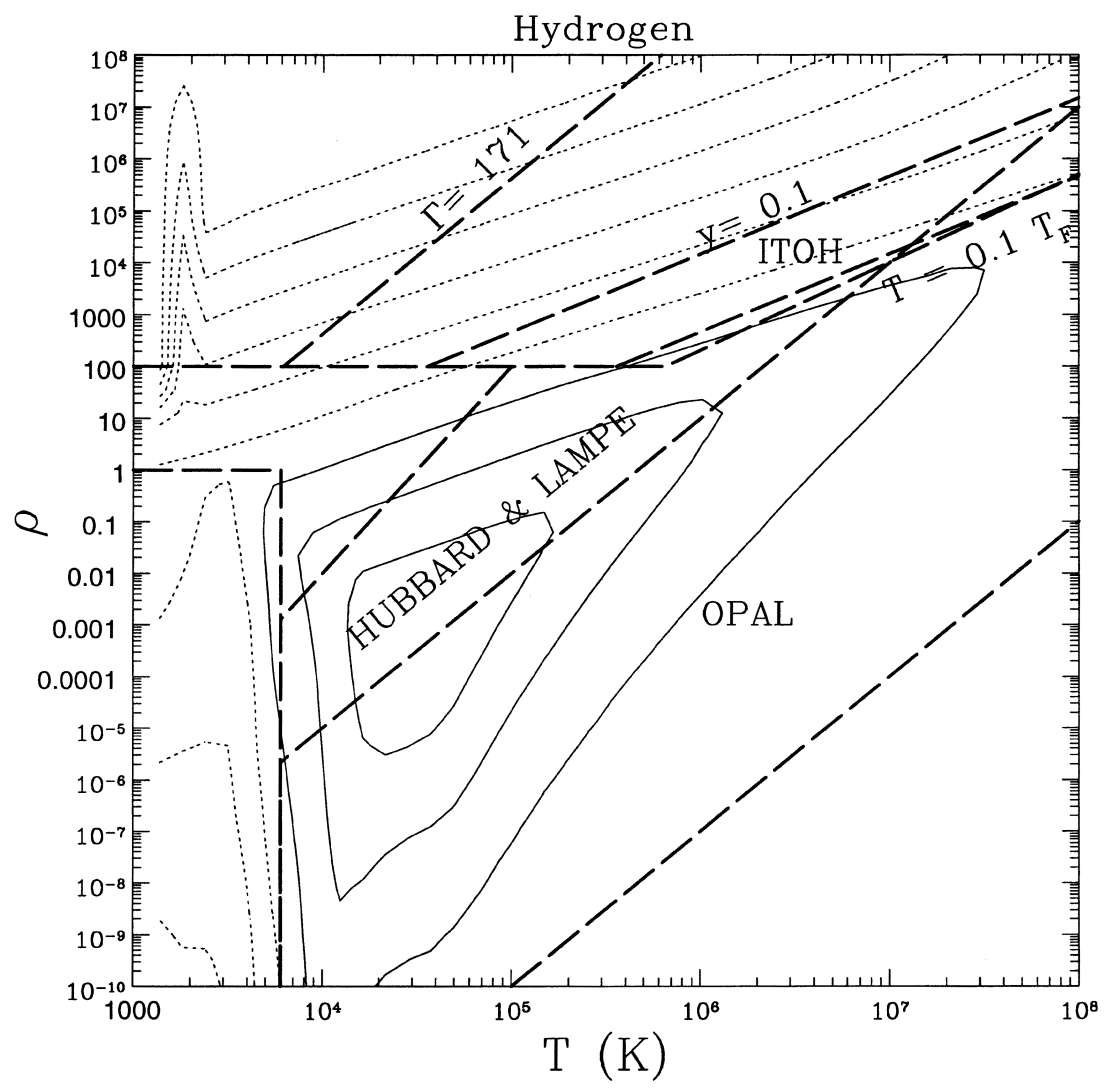

Figure 1. The hydrogen phase diagram. The thin solid lines are contours of constant $\log \kappa$, where $\kappa$ is the Rosseland mean opacity in $\mathrm{cm}^{2} \mathrm{~g}^{-1}$. The contours have values 0,2 and 4 . The dotted lines are also contours of constant $\log \kappa$ but with values -2 , -4 , etc., down to -12 (decreasing monotonically in all directions from the opacity peak near $30000 \mathrm{~K}$ and $10^{-3} \mathrm{~g} \mathrm{~cm}^{-3}$ ). The heavy dashed lines delineate regions where different tables have been used to calculate the opacity. The Itoh opacities are valid for $T<0.1 T_{\mathrm{F}}$ (where $T_{\mathrm{F}}$ is the Fermi temperature), $y<0.1$ ( $y$ measures the importance of the wave nature of the ions) and $\Gamma<171$. There is also a lower bound on the density $\rho>100 \mathrm{~g} \mathrm{~cm}^{-3}$. Outside this region we use the conductivities of Hubbard \& Lampe. The radiative opacities in the region $T \sim 10^{4}-10^{5} \mathrm{~K}$ and $\rho \sim 10^{-4}-1 \mathrm{~g} \mathrm{~cm}^{-3}$ are obtained by extrapolating the OPAL opacities to higher densities. This extrapolation is not important because the atmosphere is convective at these temperatures and densities. The box in the lower left-hand corner is the region covered by our opacity calculations. We can see the opacity minimum near $3000 \mathrm{~K}$ due to the change in the dominant opacity mechanism from $\mathrm{H}^{-}$absorption to $\mathrm{H}_{2} \mathrm{CIA}$. The strange behaviour in the upper lefthand corner is due to the extrapolation of the conductive opacities outside their range of validity. This is unimportant as no model that we consider will approach this region. 
$\mathrm{H}^{-}$ion and below by the collisionally induced opacity of $\mathrm{H}_{2}$. As noted by Bergeron, Saumon \& Wesemael (1995), the Rosseland opacity goes through a minimum near this temperature. For a pure helium atmosphere $\mathrm{He}^{-}$and Rayleigh scattering provide most of what little opacity there is.

We extend our calculations up to densities $\rho \sim 1 \mathrm{~g} \mathrm{~cm}^{-3}$. At higher densities, in lieu of an accurate calculation, we introduce abrupt pressure ionization and the opacity is determined by electron conduction at higher densities. For the case of a hydrogen atmosphere, this is unimportant because the atmosphere is sufficiently opaque that convection will dominate by the time these densities are reached. However, for the case of a helium atmosphere, this is the major source of uncertainty in our cooling times because the atmosphere is very optically thin and where we place the pressure ionization will determine the location of the photosphere.

Fig. 1 shows the contours of constant opacity in our densitytemperature parameter space for pure hydrogen. It also shows the regions where different opacity tables were used. In Fig. 2, as an example, we show the opacity as a function of temperature at $\rho=10^{-9} \mathrm{~g} \mathrm{~cm}^{-3}$ for a pure hydrogen atmosphere. There is excellent agreement between our calculation and the OPAL tables in the overlap region.

\section{THE COOLING CODE}

We have written a numerical cooling code using the Henyey method (Henyey et al. 1959; Henyey, Forbes \& Gould 1964) to solve the stellar structure equations. Our outer boundary conditions (obtained using a grey atmosphere calculation) are implemented using the Kippenhahn, Weigart \& Hoffmeister (1967b) method of triangles.

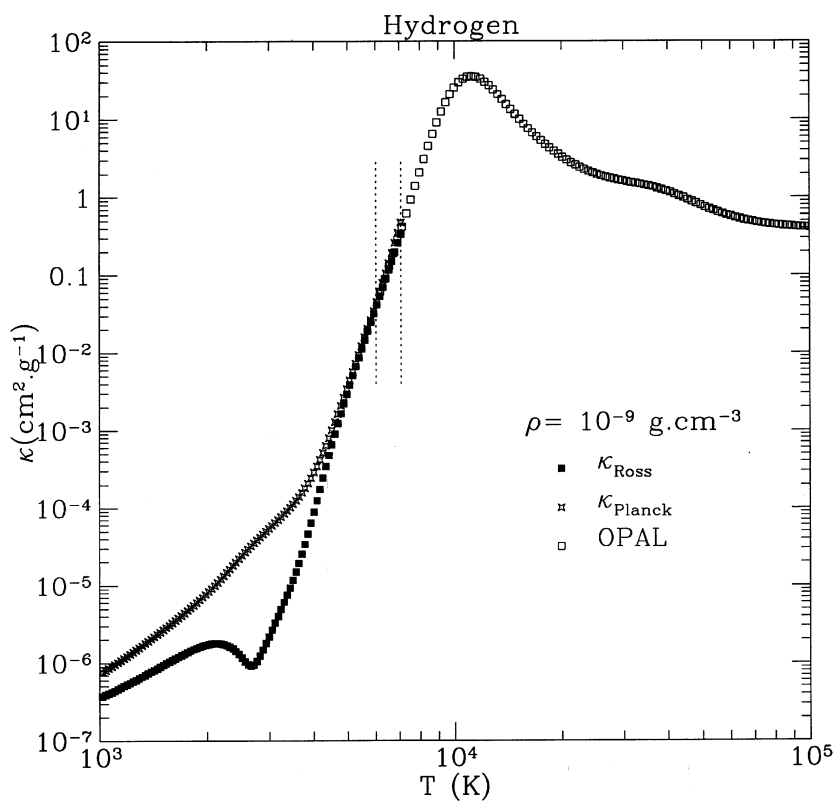

Figure 2. Matching opacities. The solid points are Rosseland mean opacities calculated using our code. The open squares are the results of the OPAL calculation. The open stars are again our calculation but showing Planck mean opacities. The vertical dotted lines delineate the region $6000-7000 \mathrm{~K}$ which is where the two calculations overlap. Once again, the minimum in the opacity near $3000 \mathrm{~K}$ is due to the change in the dominant opacity contributor, from $\mathrm{H}_{2} \mathrm{CIA}$ at lower temperatures to $\mathrm{H}^{-}$absorption at higher temperatures. We see that below $5000 \mathrm{~K}$ there is a significant discrepancy between the Planck and Rosseland mean opacities.
Apart from the opacity tables described above, we have used the hydrogen and helium equation of state of Saumon et al. (1995), supplemented by a Thomas-Fermi model where necessary (at high densities). For the carbon/oxygen sequences used in our comparisons (see Section 4.1), we have also used the carbon equation of state table of Fontaine, Graboske \& Van Horn (1977). For completeness, we also include the effects of neutrino losses using the emissivities of Itoh \& Kohyama (1983), Itoh et al. (1984) and Munakata, Kohyama \& Itoh (1985). To account for the residual nuclear burning, we incorporate the cross-sections of Fowler, Caughlan \& Zimmerman (1975). We also use the mixing length theory of convection [we tested different parametrizations, ML1, ML2 and ML3 (see Bergeron, Wesemael \& Fontaine 1992 and references therein), and found no difference in their effect on the cooling]. Appendix A briefly describes the numerical details of the calculation.

\subsection{Code tests}

The dearth of reliable helium white dwarf cooling sequences means that, in order to test our numerical method properly, we need to include carbon cores so as to test our models against those in the accepted literature. We have made comparisons with three wellknown codes, adjusting our input physics to approximate the original input physics as closely as possible. The wide variation in input physics and parameters makes this a non-trivial proposition (see Winget \& Van Horn 1987). Nevertheless, we obtain satisfactory fits that give us confidence in the accuracy of our numerical scheme and also incidentally reinforce the assertion of Winget \& Van Horn that the wide variation in published white dwarf cooling models is a result of input physics and not numerical treatment. The comparison is shown in Fig. 3. The three models are from D'Antona \& Mazzitelli (1989), Koester \& Schönberner (1986) and Wood (1992). Of particular interest is the comparison with the Wood cooling sequence because that is the most up-to-date, using $Z=0$ OPAL opacities. We do not expect agreement at late times because the calculations of the opacities for $T<6000 \mathrm{~K}$ will vary, but the agreement for ages less than a few $\times 10^{9} \mathrm{yr}$ is excellent. In particular, the arrows indicate the point at which crystallization begins, and we do not expect agreement at later times than this because our treatment of crystallization is not sufficiently detailed for these purposes (helium cores do not crystallize in a Hubble time and thus it is unimportant for this analysis). An updated version of these curves will presented in a future publication.

The one useful test of our code for the case of a helium white dwarf is a comparison with the cooling sequence of Iben \& Tutukov (1986), shown in Fig. 4. Their model was for a 0.3-M $\odot$ star which, after undergoing two hydrogen shell flashes, has a shell of $2.5 \times 10^{-4} \mathrm{M}_{\odot}$ of pure hydrogen during the white dwarf phase. They caution that their opacities are uncertain at low luminosities, but it provides a useful comparison at least for $\log L / \mathrm{L}_{\odot}>-3$. Their model also demonstrates another uncertainty for our higher luminosity models. The white dwarf stage really only begins after the end of the second hydrogen shell flash, which means that our $t=0$ corresponds to $t=10^{8} \mathrm{yr}$ in this case. The effect of this uncertainty in the starting point for the model is important for $\log L / \mathrm{L}_{\odot}>-3$.

A second uncertainty in the initial conditions is the value of the core temperature at the beginning of the evolution. Tests of our code show that an uncertainty of $3 \times 10^{7} \mathrm{~K}$ corresponds to an age uncertainty of $\sim 10^{8} \mathrm{yr}$, although the exact numbers are modeldependent. We solve this problem by using the results of evolutionary calculations (Soberman, private communication) of the 


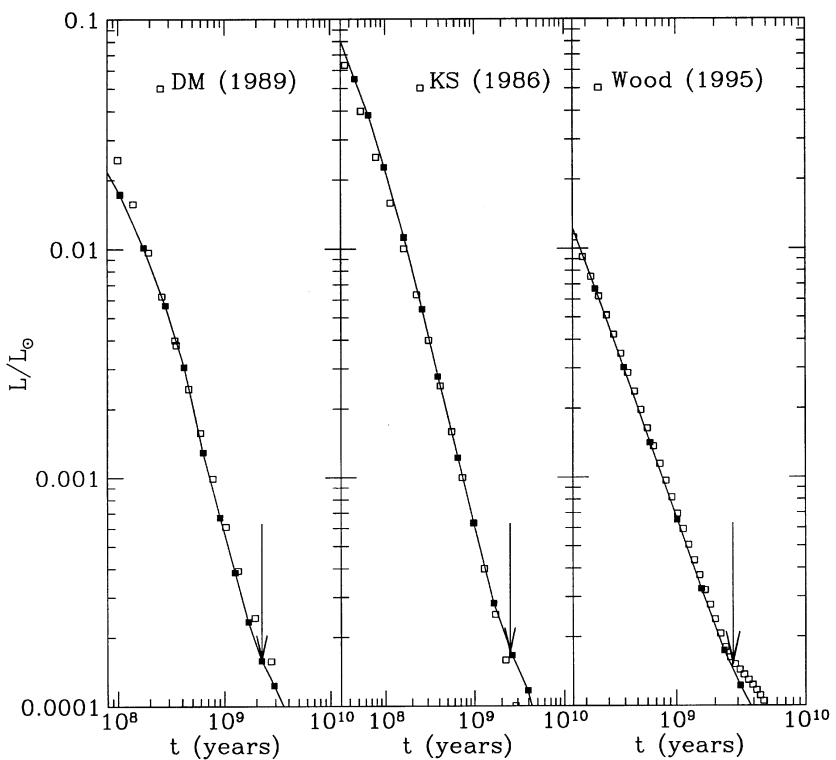

Figure 3. Code comparisons: $\mathrm{C} / \mathrm{O}$ models. The open squares denote the models that we compare against. The filled squares are our own models. The arrows denote the point at which the core of the model begins to crystallize, although some of the difference at low luminosities is due to the updated opacities. The left panel describes a $0.564-\mathrm{M}_{\odot}$ oxygen core surrounded by a helium envelope of $2.5 \times 10^{-3} \mathrm{M}_{\odot}$ and a hydrogen envelope of $3 \times 10^{-4} \mathrm{M}_{\odot}$. The metallicity is taken to be $Z=0$. The centre panel describes a $0.546-\mathrm{M}_{\odot}$ carbon core with a helium envelope of $0.022 \mathrm{M}_{\odot}$ and hydrogen envelope of $10^{-4} \mathrm{M}_{\odot}$. The metallicity is $Z=0.02$. The rightmost panel is a $0.6-\mathrm{M}_{\odot}$ star, with a carbon core, mass fraction $10^{-2}$ of helium and $10^{-4}$ of hydrogen. The metallicity is $Z=0$. We had to adjust our conductive opacities to reproduce the above results. When prior authors used Hubbard \& Lampe opacities in regions where we used Itoh opacities, we divided our opacities by 2 to compensate.

progenitors at a variety of binary separations around a neutron star, using the stellar evolution code of Eggleton (1971) (see also Pols et al. 1995). This provides us with an estimate of the initial central temperature for a given remnant mass, which we use as a starting point for our calculations. For most of the systems that we address in Paper II, with ages $>1$ Gyr, this uncertainty is unimportant.

There are also uncertainties in the evolution associated with the uncertainties in the input physics. We investigated the effect of extrapolating the Itoh opacities to lower densities instead of using the old Hubbard \& Lampe opacities for those regions. This was found to have little impact on the cooling sequences, because the conductivity is most important in the highest density regions, which are covered by the Itoh tables. A second uncertainty is the effect of extrapolating the OPAL opacities to higher densities in the region $T \sim 10^{4}-10^{5} \mathrm{~K}$ and $\rho \sim 10^{-3}-1 \mathrm{~g} \mathrm{~cm}^{-3}$. This is not terribly important because the atmosphere is convective in this region, and so the extrapolation will affect only the depth at which convection terminates.

A major uncertainty of past calculations was for the lowtemperature $(T<6000 \mathrm{~K})$ opacities. Our calculations in Section 3 are designed to solve this problem, at least for the hydrogen atmospheres. The problem persists with the helium atmospheres, where the photospheric opacity is determined by the pressure ionization of helium, in a regime where accurate opacity calculations do not exist. For some models, we also have convective mixing of the elements. We assume that the convective zone is

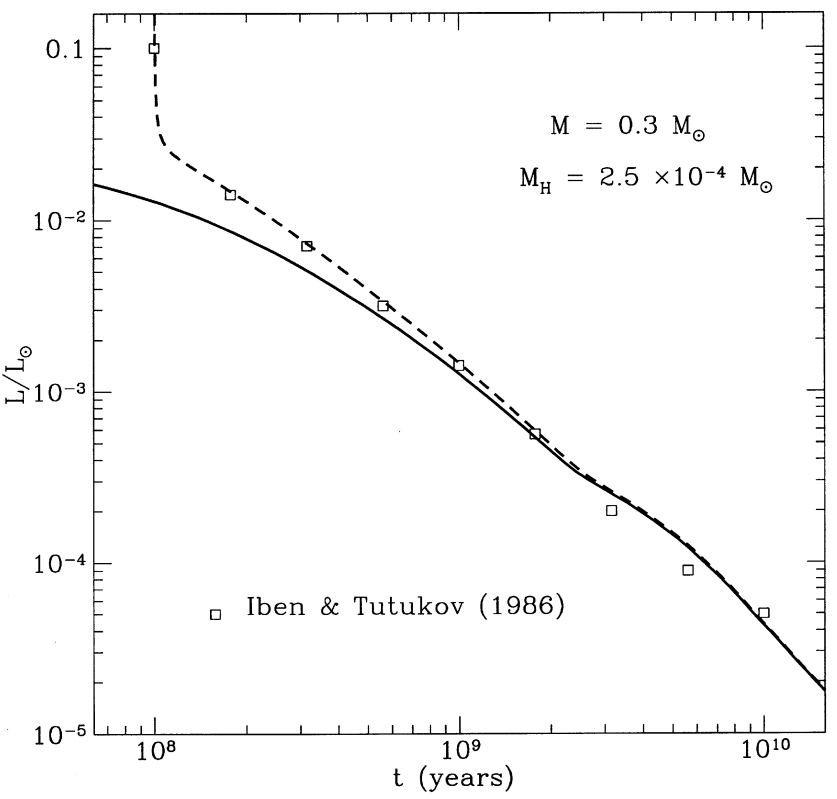

Figure 4. Code comparisons: He model. The solid line shows our model with no corrections for different starting points. The dashed line corresponds to the same model, but with the age incremented by $10^{8} \mathrm{yr}$, to compensate for the time spent in prior evolutionary stages. The open squares are the results of Iben \& Tutukov (1986). The agreement is excellent until $t \sim 2$ Gyr, by which point Iben $\&$ Tutukov caution that their opacities are uncertain.

mixed to a uniform composition determined by the relative mass fractions of hydrogen and helium in the convective zone. When the convective zone retreats from the lower layers, we assume that the separation is instantaneous, i.e. at all times, the composition in the convective zone is determined by the depth of the convective base (i.e. the mass of helium in the convection zone relative to the hydrogen envelope mass). The admixture of helium into the hydrogen atmosphere does not restrict the validity of our opacity calculations at low temperatures. Because of the extremely low neutral helium opacities, 5 per cent of hydrogen by mass is still sufficient to provide enough opacity that the photospheric pressure lies well below the pressure ionization value.

\section{RESULTS}

In this section, we will describe in detail the cooling of a $0.3-\mathrm{M}_{\odot}$ helium core star with a thick $\left(3 \times 10^{-4} \mathrm{M}_{\odot}\right)$ hydrogen envelope, shown in Figs 5-7. We start our models with an age of $10^{8} \mathrm{yr}$ (the time taken for the Iben \& Tutukov models to reach the end of the last shell flash). We find that, even for these hydrogen masses and at early ages, the residual nuclear burning contribution to the luminosity is never more than $\sim 1$ per cent (see Fig. 5). The neutrino luminosity is never more than $\sim 10$ per cent of the photon luminosity. Thus over the first few $\times 10^{6} \mathrm{yr}$ the star completes the contraction to the white dwarf configuration that it began when the last shell flash ended (see, e.g., Iben \& Tutukov 1986 , table 1). In these early stages, the helium core is only mildly degenerate (central degeneracies $\sim 30$ ) with $\eta \sim 1$ at the hydrogen-helium boundary, although the core is already approaching an isothermal state (0.99 of the stellar mass has $T>0.5 T_{\mathrm{c}}$ ). The hydrogen envelope is entirely radiative at this stage, so that this is the time at which the star most resembles the Mestel ideal (see Fig. 5). 


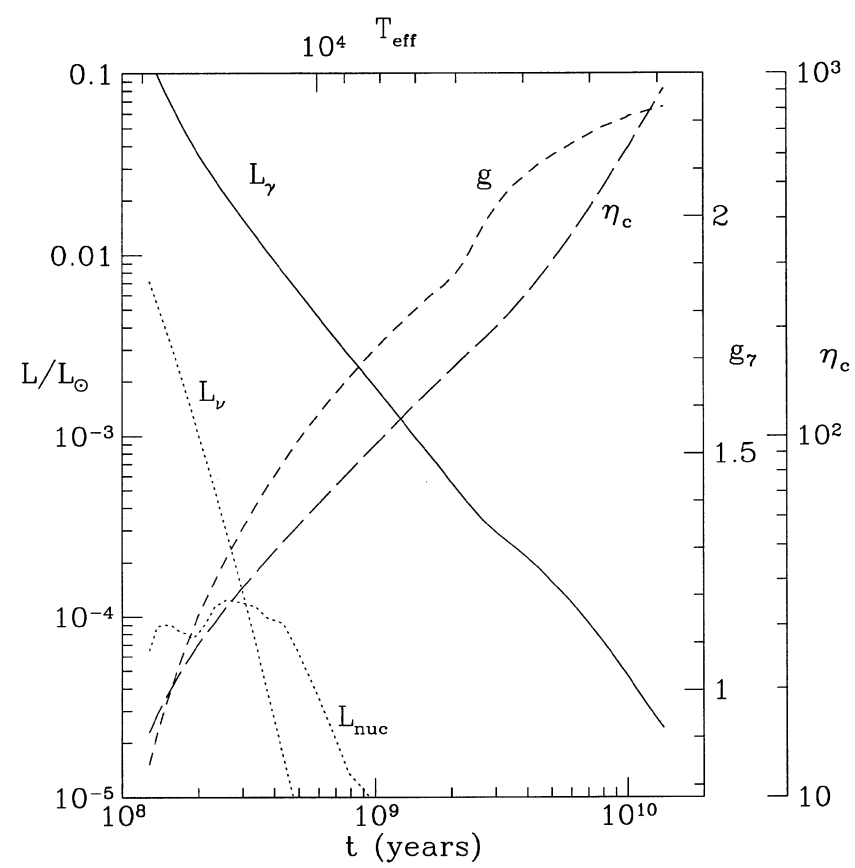

Figure 5. Cooling of a $0.3-\mathrm{M}_{\odot}$ model. We show here the cooling of a 0.3$\mathrm{M}_{\odot}$ star with a hydrogen envelope of $3 \times 10^{-4} \mathrm{M}_{\odot}$. The solid line is the electromagnetic bolometric luminosity $L_{\gamma}$, the dotted lines indicate the neutrino and nuclear luminosities (the nuclear contribution is included in the bolometric luminosity) and the short- and long-dashed lines indicate the gravity (in units of $10^{7} \mathrm{~cm} \mathrm{~s}^{-2}$ ) and the central degeneracy $\left(\eta_{\mathrm{c}}=E_{\mathrm{F}} / k T\right)$ respectively. The effective temperatures corresponding to the various ages for this model are shown on the top axis, ranging from $20000 \mathrm{~K}$ at the left to $3000 \mathrm{~K}$ at the right.

When $T_{\text {eff }} \sim 15000 \mathrm{~K}\left(\sim 3 \times 10^{8} \mathrm{yr}\right)$, a small convective zone appears near the surface. This zone remains relatively thin until $T_{\text {eff }} \sim 10^{4} \mathrm{~K}\left(\sim 10^{9} \mathrm{yr}\right)$, when it starts to deepen as a consequence of the movement of the hydrogen ionization zone to greater depths (Fig. 7). The recombination of hydrogen also leads to an increase in the photospheric density (since neutral hydrogen has a smaller opacity than ionized hydrogen). This increase is only halted once molecular hydrogen begins to form $\left(T_{\mathrm{eff}} \sim 5000 \mathrm{~K}, t \sim 4 \times 10^{9} \mathrm{yr}\right.$, see Fig. 6). These photospheric changes also lead to the flattening of the cooling curves and the increase in the gravity seen in Figs 10 and 15 (later), similar to effects in $\mathrm{C} / \mathrm{O}$ models as described in D'Antona \& Mazzitelli (1990). The deepening of the convective zone continues until the base reaches a depth where the conduction due to degenerate electrons is more efficient than convection. This occurs after $3 \times 10^{9} \mathrm{yr}$. At its deepest extent, the convective zone contains $\sim 5 \times 10^{-5} \mathrm{M}_{\odot}$. This is only a couple of scaleheights above the hydrogen-helium interface, so we might expect some small amount of photospheric helium contamination (since the convection extends to optical depths $\tau \sim 0.3$ ) below $T_{\text {eff }} \sim 6000 \mathrm{~K}$ (see also Böhm et al. 1977). However, the helium will be present only in trace amounts and will not affect the cooling. As the star cools further, it will revert again to a pure hydrogen atmosphere because of the formation of a radiative buffer zone near the surface below $T_{\text {eff }} \sim 3500 \mathrm{~K}$. This occurs because there is a minimum in the atmospheric opacity as the primary opacity contribution changes from CIA of $\mathrm{H}_{2}$ to $\mathrm{H}^{-}$ absorption. If this minimum value is low enough, the region of the minimum will be radiative, but convection will continue to operate both above and below. This would allow the surface

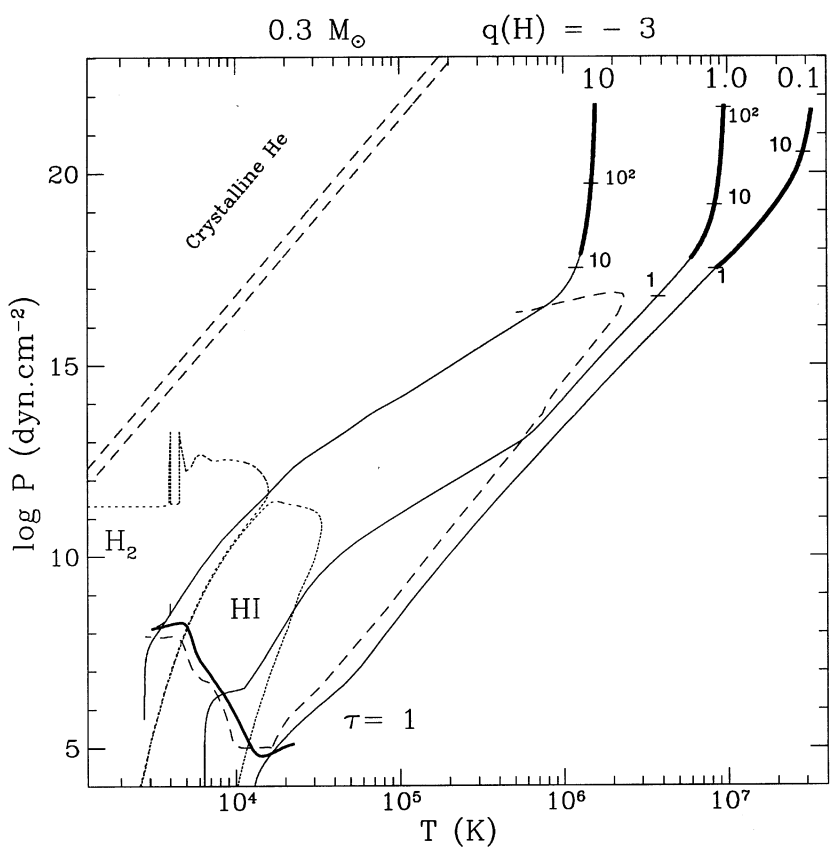

Figure 6. Evolution in the phase diagram. This $T-P$ phase diagram shows three representative atmosphere profiles (labelled at the top by their age in Gyr) for the evolution of the same model as shown in Fig. 5. The heavy solid lines indicate the helium parts of the star and the thin solid lines indicate the hydrogen part. The dotted lines delineate the regions of 50-50 division between $\mathrm{H}_{\mathrm{I}}-\mathrm{H}_{\mathrm{II}}$ and $\mathrm{H}_{2}-\mathrm{H}_{\text {I }}$ respectively. The dashed line indicates the boundary of the convective region for this model (the other pair of dashed lines in the upper left-hand corner indicates the crystallization boundary of helium). The thick solid line at the lower left indicates the location of the photosphere for this cooling sequence. The labelled tick marks on each of the three curves indicate the points at which the degeneracy parameter $\eta=E_{\mathrm{F}} / k T$ has that particular value.

helium to diffuse out of the top convection zone, leaving the atmosphere once again in a pure hydrogen state.

The features of the above sequence are common to most of the models we discuss here. The primary differences occur at the lower mass end of the models $\left(M \sim 0.15 \mathrm{M}_{\odot}\right)$. The lower masses lead to lower central densities and lower degeneracy, so that the model radii are somewhat larger (hence with lower gravities) and convection extends deeper into the cooler models (because conduction is less efficient). Thus, even for the thickest hydrogen envelopes considered here, ${ }^{1}$ the $0.15-\mathrm{M}_{\odot}$ models do dredge up some helium, becoming mixed atmosphere stars for a short while until the base of the convective zone retreats again. Fig. 8 shows a diagram similar to Fig. 7 but for a $0.15-\mathrm{M}_{\odot}$ model. We see that the base of the convective zone penetrates to the helium layer, leading to an atmospheric helium abundance of $\sim 15$ per cent in this case. Hence, for about $1.5 \mathrm{Gyr}$, the star would exhibit the characteristics of a cool $\mathrm{H} / \mathrm{He}$ atmosphere star, although, with a temperature of $4000-5000 \mathrm{~K}$, it would be difficult to identify it as such. For the thick hydrogen layer models, only the lowest mass models are

\footnotetext{
${ }^{1}$ We have used the upper limit derived by Iben \& Tutukov (1986), although it is likely to increase somewhat for smaller core masses. It is important to note at this point that many stellar evolution calculations such as those by Pylyser \& Savonije (1988) and Alberts et al. (1996) cannot be used to set this limit, because they make use of a stellar evolution code that cannot resolve the shell flashes described by Iben \& Tutukov.
} 


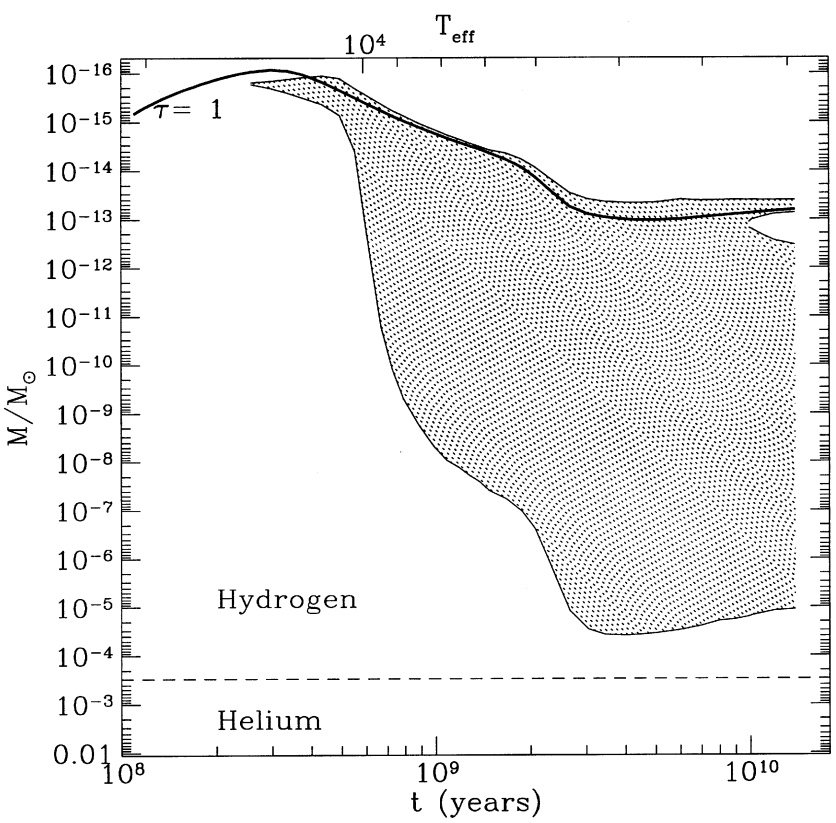

Figure 7. 0.3-M $\mathrm{M}_{\odot}$ convective zone. Here we show the mass in the convective zone as a function of age (or effective temperature). The shaded region is the convective zone, while the heavy solid line indicates the location of the photosphere. The dashed line indicates the hydrogen-helium interface in this set of models. We note the appearance of a radiative buffer zone at late times associated with the transition from $\mathrm{H}^{-}$opacity to $\mathrm{H}_{2}$ opacity.

affected by atmospheric helium contamination. In the sequence of models with thin $\left(\sim 10^{-6} \mathrm{M}_{\odot}\right)$ hydrogen layers, dredge-up occurs for all masses. In Fig. 9 we show the effective temperature ranges in which this occurs for all model masses.

Figs 10 and 11 show the cooling sequences for six different models spanning the range of representative masses. We show curves for 0.15 , 0.25 and $0.45 \mathrm{M}_{\odot}$, with two different hydrogen envelope masses, $3 \times 10^{-4} \mathrm{M}_{\odot}$ (thick) and $10^{-6} \mathrm{M}_{\odot}$ (thin). For completeness we also show the sequences for pure helium models in Fig. 12. In principle, as found by D'Antona \& Mazzitelli (1989), one can obtain shorter cooling times for these models, but the opacities for $T_{\text {eff }}<6000 \mathrm{~K}$ are rather uncertain, and thus we do not expect the cooling curves to be accurate at temperatures below this value.

In order to make comparison with observations easier, we have also calculated the blackbody absolute magnitudes for these sequences, using the flux calibrations of Bessell (1979). The most common bands used are $V$ and $I$. By comparison with the detailed atmospheric calculations of Bergeron et al. (1995), we find that this is an excellent approximation for $T_{\text {eff }}>4500 \mathrm{~K}$. This can be understood by comparing the Rosseland and Planck mean opacities shown in Fig. 2 which correspond to different frequency weightings of the true monochromatic opacity. When they are equal this indicates an approximately uniform continuum opacity and the blackbody approximation is valid. For $T_{\text {eff }}<4500 \mathrm{~K}$, the CIA of $\mathrm{H}_{2}$ begins to dominate and this leads to significant deviations from the blackbody approximation, as can be seen in Fig. 2. Although the Bergeron et al. results are calculated for higher gravities than we require, they represent an upper limit on the deviations from a blackbody. This is because CIA is an intrinsically high-density phenomenon (it requires collisional interactions) and photospheric densities are lower in lower gravity atmospheres. This can be demonstrated by comparing the low-temperature results for the $\log g=7.5$ and $\log g=8.5$ atmospheres of Bergeron et al. We will

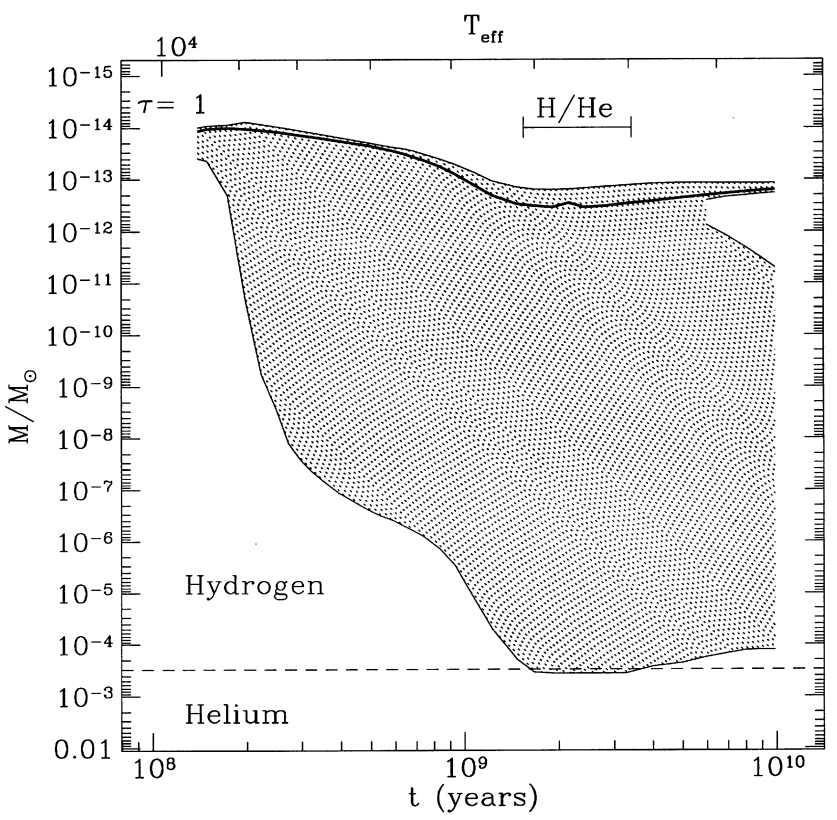

Figure 8. $0.15-\mathrm{M}_{\odot}$ convective zone. Once again the shaded region is the convective zone, and the heavy solid line denotes the position of the photosphere. We note that, for $T_{\text {eff }} \sim 4000-5000 \mathrm{~K}$, the atmosphere will be contaminated with helium.

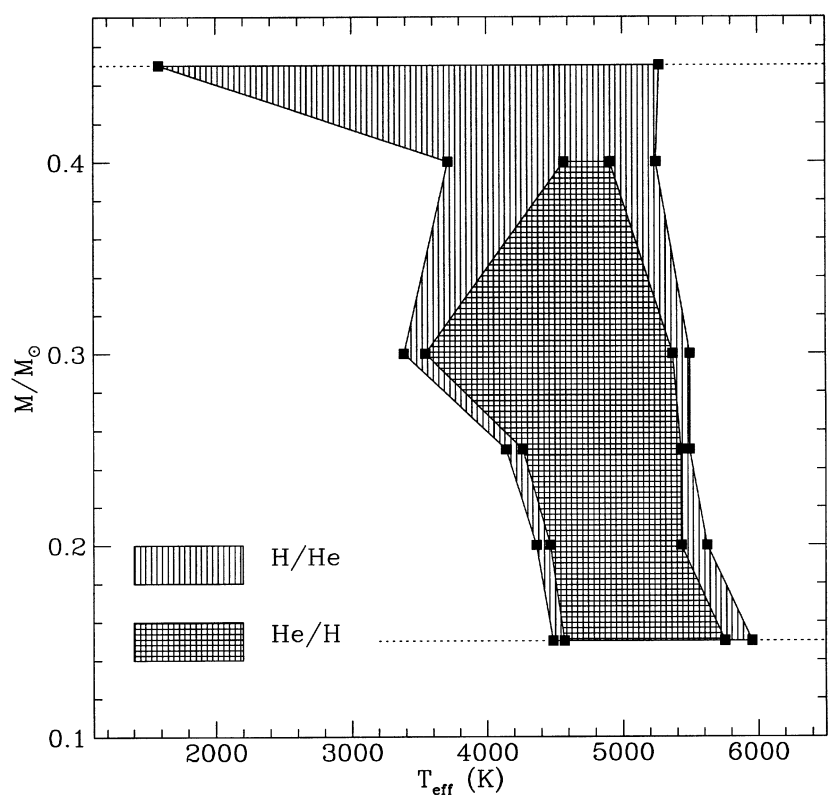

Figure 9. Atmospheric helium contamination for thin hydrogen envelopes. The shaded regions indicate those models in which the convective zone extends into the helium layer and thus causes atmospheric helium contamination. We consider two representative cases. The area marked as $\mathrm{H} / \mathrm{He}$ is characterized by a mass fraction of helium $X_{\mathrm{He}}>0.1$. The area marked as $\mathrm{He} / \mathrm{H}$ is characterized by $X_{\mathrm{He}}>0.8$. This cut-off value may seem rather high, but we note that the dredge-up occurs for temperatures at which helium is neutral and thus makes little contribution to the opacity. The horizontal dotted lines indicate the mass limits of the models we calculated, so that the extent of the convective regions outside these bounds is unknown. These models are for a hydrogen envelope of mass $M_{\mathrm{H}}=10^{-6} \mathrm{M}_{\odot}$. 


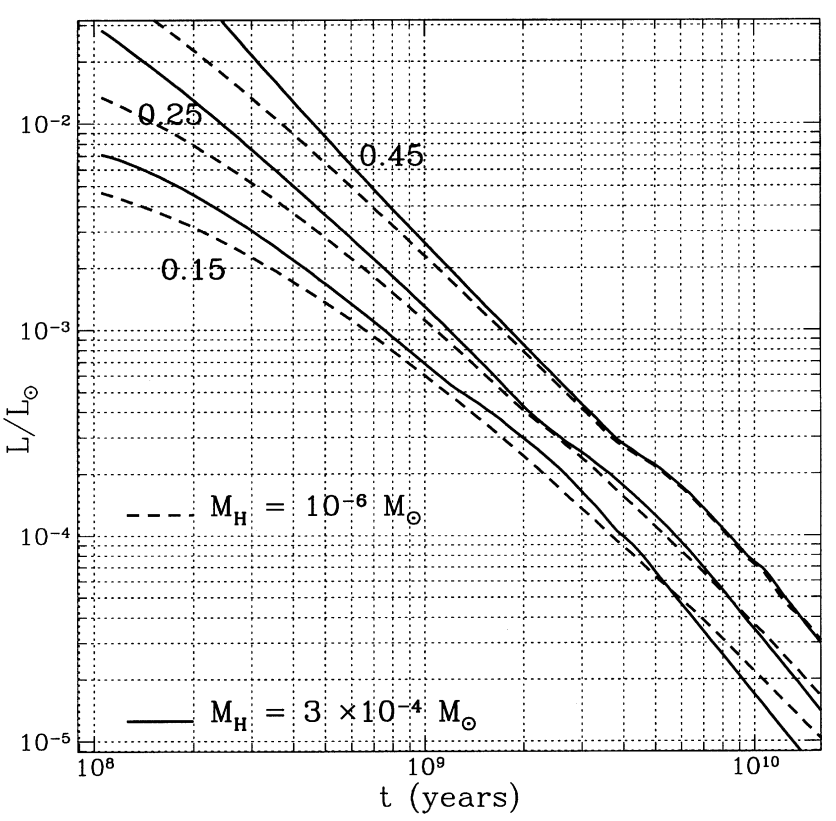

Figure 10. Hydrogen cooling sequences -1 : luminosity evolution. The solid lines indicate model white dwarf cooling sequences with a hydrogen envelope of $3 \times 10^{-4} \mathrm{M}_{\odot}$ for each of three representative total masses. The dashed lines are the equivalent sequences with a smaller hydrogen envelope of $10^{-6} \mathrm{M}_{\odot}$. The difference in luminosities at earlier times is a result of the thicker hydrogen layer leading to a larger stellar radius (the effective temperatures are closer - see Fig. 11).

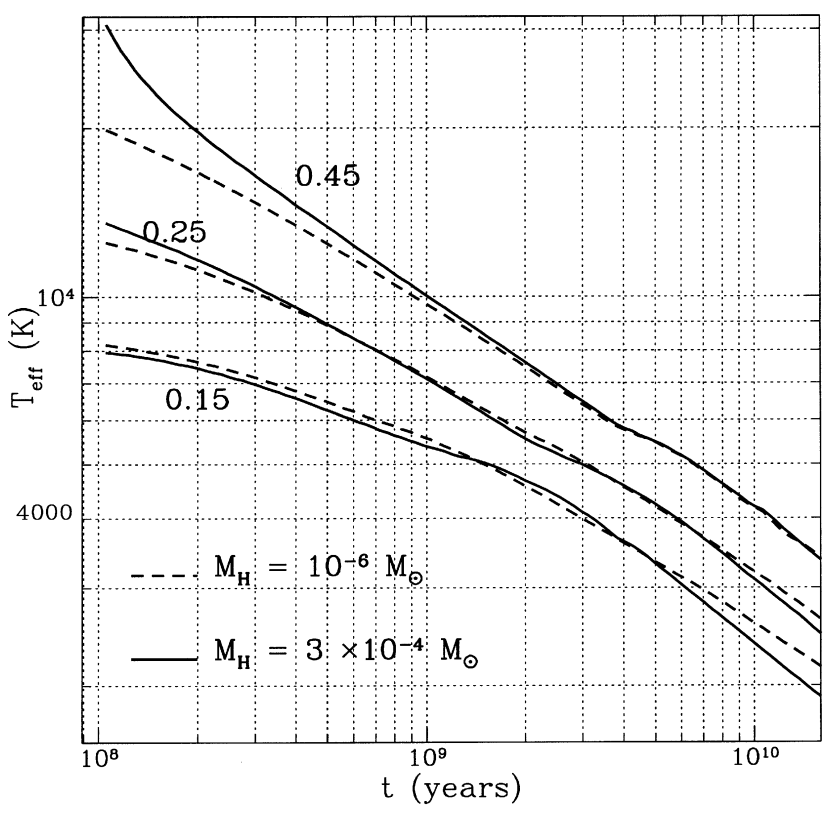

Figure 11. Hydrogen cooling sequences - 2: temperature evolution. Here we show the effective temperature for the same sequences as in Fig. 10. Note the large variation in temperature with envelope mass for the most massive models. This is the effect of the contribution of residual hydrogen burning at the base of the thicker hydrogen envelope.
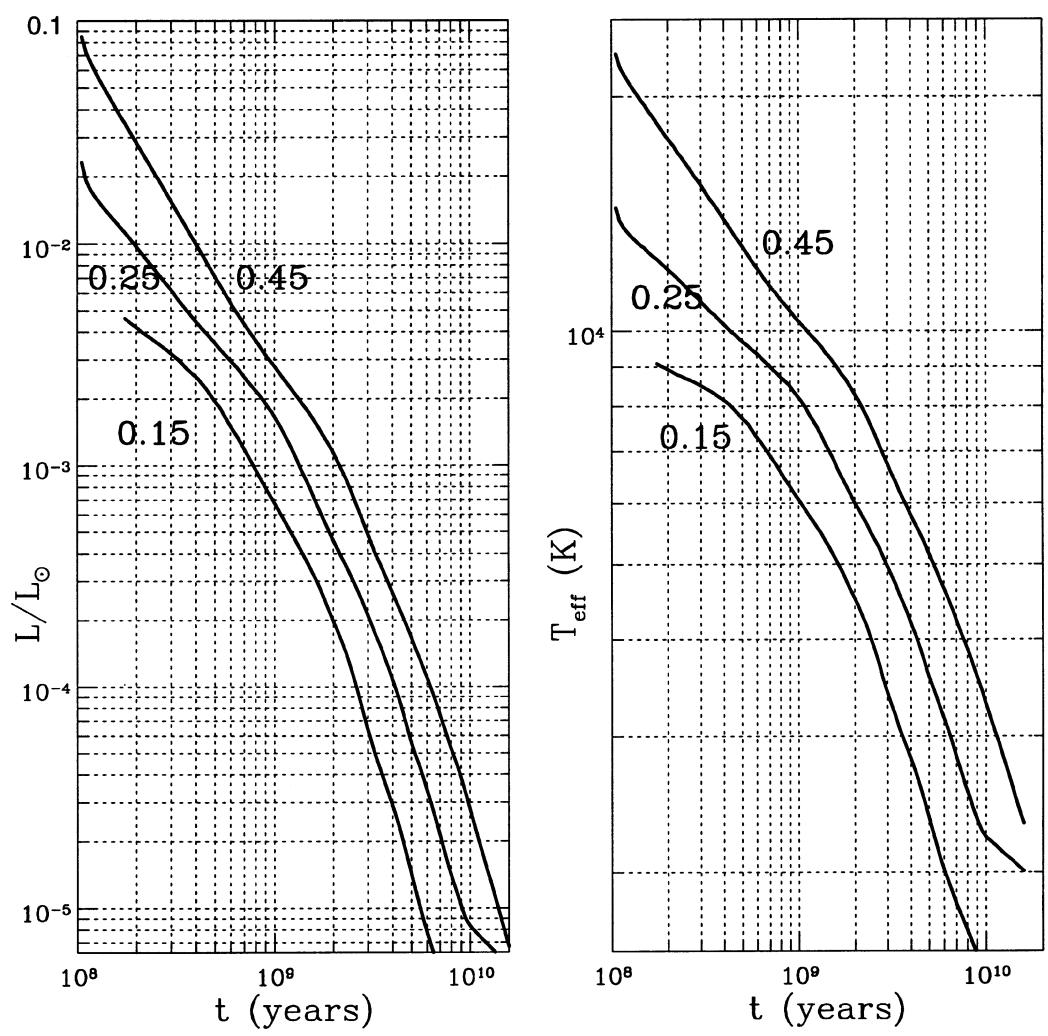

Figure 12. Helium cooling sequences. We show here pure helium models for the same masses as before. The evolution at effective temperatures below $6000 \mathrm{~K}$ is uncertain because of the inaccuracy of the photospheric opacities for neutral helium at these temperatures. 
Table 1. Masses of helium core white dwarfs $\left(\mathrm{M}_{\odot}\right)$.

\begin{tabular}{|c|c|c|c|c|c|c|c|c|c|c|c|c|c|c|c|c|}
\hline $\begin{array}{l}T_{\text {eff }} \\
(\mathrm{K})\end{array}$ & 10.0 & 10.5 & 11.0 & 11.5 & 12.0 & 12.5 & 12.75 & 13.0 & $\begin{array}{c}M_{I} \\
13.25\end{array}$ & 13.5 & 13.75 & 14.0 & 14.25 & 14.50 & 14.75 & 15.0 \\
\hline 12000 & 0.27 & 0.36 & & & & & & & & & & & & & & \\
\hline 11500 & 0.25 & 0.33 & 0.45 & & & & & & & & & & & & & \\
\hline 11000 & 0.24 & 0.30 & 0.43 & & & & & & & & & & & & & \\
\hline 10500 & 0.22 & 0.28 & 0.40 & & & & & & & & & & & & & \\
\hline 10000 & 0.21 & 0.26 & 0.36 & & & & & & & & & & & & & \\
\hline 9500 & 0.19 & 0.24 & 0.32 & 0.45 & & & & & & & & & & & & \\
\hline 9000 & 0.18 & 0.22 & 0.29 & 0.42 & & & & & & & & & & & & \\
\hline 8500 & 0.17 & 0.20 & 0.26 & 0.38 & & & & & & & & & & & & \\
\hline 8000 & 0.16 & 0.18 & 0.23 & 0.32 & & & & & & & & & & & & \\
\hline 7500 & & 0.16 & 0.20 & 0.27 & 0.42 & & & & & & & & & & & \\
\hline 7000 & & & 0.18 & 0.23 & 0.34 & & & & & & & & & & & \\
\hline 6500 & & & 0.15 & 0.19 & 0.27 & 0.43 & & & & & & & & & & \\
\hline 6000 & & & & 0.16 & 0.21 & 0.33 & 0.42 & & & & & & & & & \\
\hline 5800 & & & & & 0.19 & 0.29 & 0.37 & 0.44 & & & & & & & & \\
\hline 5600 & & & & & 0.17 & 0.25 & 0.32 & 0.41 & & & & & & & & \\
\hline 5400 & & & & & 0.15 & 0.21 & 0.27 & 0.35 & 0.44 & & & & & & & \\
\hline 5200 & & & & & & 0.18 & 0.22 & 0.29 & 0.39 & & & & & & & \\
\hline 5000 & & & & & & & 0.19 & 0.24 & 0.32 & 0.42 & & & & & & \\
\hline 4800 & & & & & & & 0.15 & 0.20 & 0.26 & 0.34 & 0.44 & & & & & \\
\hline 4600 & & & & & & & & 0.16 & 0.20 & 0.27 & 0.37 & & & & & \\
\hline 4400 & & & & & & & & & 0.16 & 0.21 & 0.28 & 0.39 & & & & \\
\hline 4200 & & & & & & & & & & 0.16 & 0.22 & 0.29 & 0.40 & & & \\
\hline 4000 & & & & & & & & & & & 0.16 & 0.22 & 0.29 & 0.40 & & \\
\hline 3800 & & & & & & & & & & & & 0.16 & 0.21 & 0.29 & 0.40 & \\
\hline 3600 & & & & & & & & & & & & & & 0.20 & 0.27 & 0.37 \\
\hline 3400 & & & & & & & & & & & & & & & 0.18 & 0.24 \\
\hline
\end{tabular}

The values in this table are for a thick hydrogen envelope $\left(3 \times 10^{-4} \mathrm{M}_{\odot}\right)$.

Table 2. Ages of helium core white dwarfs (Gyr).

\begin{tabular}{|c|c|c|c|c|c|c|c|c|c|c|c|c|c|c|c|c|}
\hline $\begin{array}{l}T_{\text {eff }} \\
(\mathrm{K})\end{array}$ & 10.0 & 10.5 & 11.0 & 11.5 & 12.0 & 12.5 & 12.75 & 13.0 & $\begin{array}{c}M_{I} \\
13.25\end{array}$ & 13.5 & 13.75 & 14.0 & 14.25 & 14.5 & 14.75 & 15.0 \\
\hline 12000 & 0.2 & 0.4 & & & & & & & & & & & & & & \\
\hline 11500 & 0.2 & 0.4 & 0.7 & & & & & & & & & & & & & \\
\hline 11000 & 0.2 & 0.4 & 0.8 & & & & & & & & & & & & & \\
\hline 10500 & 0.2 & 0.4 & 0.8 & & & & & & & & & & & & & \\
\hline 10000 & 0.2 & 0.4 & 0.7 & & & & & & & & & & & & & \\
\hline 9500 & 0.1 & 0.4 & 0.7 & 1.1 & & & & & & & & & & & & \\
\hline 9000 & 0.1 & 0.3 & 0.6 & 1.2 & & & & & & & & & & & & \\
\hline 8500 & 0.1 & 0.3 & 0.6 & 1.1 & & & & & & & & & & & & \\
\hline 8000 & 0.1 & 0.3 & 0.6 & 1.1 & & & & & & & & & & & & \\
\hline 7500 & & 0.3 & 0.5 & 1.0 & 1.8 & & & & & & & & & & & \\
\hline 7000 & & & 0.5 & 0.9 & 1.6 & & & & & & & & & & & \\
\hline 6500 & & & 0.4 & 0.8 & 1.5 & 2.6 & & & & & & & & & & \\
\hline 6000 & & & & 0.7 & 1.3 & 2.3 & 3.1 & & & & & & & & & \\
\hline 5800 & & & & & 1.2 & 2.1 & 2.9 & 3.9 & & & & & & & & \\
\hline 5600 & & & & & 1.1 & 2.0 & 2.6 & 3.7 & & & & & & & & \\
\hline 5400 & & & & & 1.0 & 1.8 & 2.4 & 3.4 & 4.9 & & & & & & & \\
\hline 5200 & & & & & & 1.6 & 2.2 & 3.1 & 4.6 & & & & & & & \\
\hline 5000 & & & & & & & 2.0 & 2.8 & 4.0 & 5.9 & & & & & & \\
\hline 4800 & & & & & & & 1.8 & 2.5 & 3.5 & 5.1 & 7.1 & & & & & \\
\hline 4600 & & & & & & & & 2.2 & 3.1 & 4.4 & 6.4 & & & & & \\
\hline 4400 & & & & & & & & & 2.7 & 3.7 & 5.3 & 7.7 & & & & \\
\hline 4200 & & & & & & & & & & 3.1 & 4.3 & 6.1 & 8.9 & & & \\
\hline 4000 & & & & & & & & & & & 3.4 & 4.8 & 6.9 & 10.0 & & \\
\hline 3800 & & & & & & & & & & & & 3.7 & 5.3 & 7.6 & 10.9 & \\
\hline 3600 & & & & & & & & & & & & & & 5.5 & 7.9 & 11.3 \\
\hline 3400 & & & & & & & & & & & & & & & 5.5 & 7.9 \\
\hline
\end{tabular}

The values in this table are for a thick hydrogen envelope $\left(3 \times 10^{-4} \mathrm{M}_{\odot}\right)$. 
Table 3. Gravities of helium core white dwarfs $(\log g)$.

\begin{tabular}{|c|c|c|c|c|c|c|c|c|c|c|c|c|c|c|c|c|}
\hline $\begin{array}{l}T_{\text {eff }} \\
(\mathrm{K})\end{array}$ & 10.0 & 10.5 & 11.0 & 11.5 & 12.0 & 12.5 & 12.75 & 13.0 & $\begin{array}{c}M_{I} \\
13.25\end{array}$ & 13.5 & 13.75 & 14.0 & 14.25 & 14.5 & 14.75 & 15.0 \\
\hline 12000 & 7.01 & 7.33 & & & & & & & & & & & & & & \\
\hline 11500 & 6.95 & 7.27 & 7.60 & & & & & & & & & & & & & \\
\hline 11000 & 6.88 & 7.19 & 7.54 & & & & & & & & & & & & & \\
\hline 10500 & 6.81 & 7.12 & 7.47 & & & & & & & & & & & & & \\
\hline 10000 & 6.74 & 7.04 & 7.38 & & & & & & & & & & & & & \\
\hline 9500 & 6.66 & 6.95 & 7.28 & 7.62 & & & & & & & & & & & & \\
\hline 9000 & 6.57 & 6.85 & 7.18 & 7.55 & & & & & & & & & & & & \\
\hline 8500 & 6.50 & 6.75 & 7.07 & 7.44 & & & & & & & & & & & & \\
\hline 8000 & 6.39 & 6.65 & 6.95 & 7.30 & & & & & & & & & & & & \\
\hline 7500 & & 6.53 & 6.82 & 7.16 & 7.54 & & & & & & & & & & & \\
\hline 7000 & & & 6.68 & 7.00 & 7.37 & & & & & & & & & & & \\
\hline 6500 & & & 6.53 & 6.83 & 7.18 & 7.57 & & & & & & & & & & \\
\hline 6000 & & & & 6.65 & 6.97 & 7.35 & 7.56 & & & & & & & & & \\
\hline 5800 & & & & & 6.87 & 7.25 & 7.45 & 7.64 & & & & & & & & \\
\hline 5600 & & & & & 6.78 & 7.13 & 7.34 & 7.55 & & & & & & & & \\
\hline 5400 & & & & & 6.67 & 7.01 & 7.22 & 7.43 & 7.62 & & & & & & & \\
\hline 5200 & & & & & & 6.88 & 7.07 & 7.29 & 7.51 & & & & & & & \\
\hline 5000 & & & & & & & 6.93 & 7.14 & 7.36 & 7.58 & & & & & & \\
\hline 4800 & & & & & & & 6.77 & 6.98 & 7.20 & 7.42 & 7.63 & & & & & \\
\hline 4600 & & & & & & & & 6.81 & 7.03 & 7.25 & 7.48 & & & & & \\
\hline 4400 & & & & & & & & & 6.84 & 7.07 & 7.29 & 7.52 & & & & \\
\hline 4200 & & & & & & & & & & 6.86 & 7.08 & 7.31 & 7.55 & & & \\
\hline 4000 & & & & & & & & & & & 6.86 & 7.09 & 7.32 & 7.56 & & \\
\hline 3800 & & & & & & & & & & & & 6.84 & 7.07 & 7.30 & 7.55 & \\
\hline 3600 & & & & & & & & & & & & & & 7.02 & 7.26 & 7.50 \\
\hline 3400 & & & & & & & & & & & & & & & 6.94 & 7.18 \\
\hline
\end{tabular}

The values in this table are for a thick hydrogen envelope $\left(3 \times 10^{-4} \mathrm{M}_{\odot}\right)$.

discuss the uncertainties again in Paper II when we come to apply these models.

In Tables 1, 2 and 3 we present the model parameters (mass, age and gravity) as a function of the observable quantities $T_{\text {eff }}$ and $M_{I}$. Each entry contains three numbers, the mass in $\mathrm{M}_{\odot}$, the age in $\mathrm{Gyr}$ and $\log g$. Figs 13 and 14 show $M_{V}$ and $M_{I}$ as a function of age.

For stars with hydrogen envelopes and effective temperatures $>7000 \mathrm{~K}$, spectroscopic determinations of effective temperature and gravity can provide a direct measurement of the white dwarf mass (Bergeron, Wesemael \& Fontaine 1991), provided that one has a relationship between radius and mass. We have calculated the mass-radius relations for both our thick and thin hydrogen layer models for the full range of helium core white dwarf masses. We fit these by a relation between gravity $g=G M / R^{2}$ and effective temperature $T_{\text {eff }}$ for a given mass, namely

$g=F\left(T_{\text {eff }}\right)\left(a_{2}-a_{3} T_{\text {eff }}\right)$,

with

$F\left(T_{\text {eff }}\right)=1+\frac{a_{1} T_{\text {eff }}}{1+9 \exp \left[4 \times 10^{-3}\left(T_{\text {eff }}-5800 \mathrm{~K}\right)\right]}$,

and

$a_{1}=9.91 \times 10^{-7}\left(\frac{M}{\mathrm{M}_{\odot}}\right)^{-2.33}$,

$a_{2}=1.69 \times 10^{8}\left(\frac{M}{\mathrm{M}_{\odot}}\right)^{1.68}$,

$a_{3}=946\left(\frac{M}{\mathrm{M}_{\odot}}\right)^{0.17}$

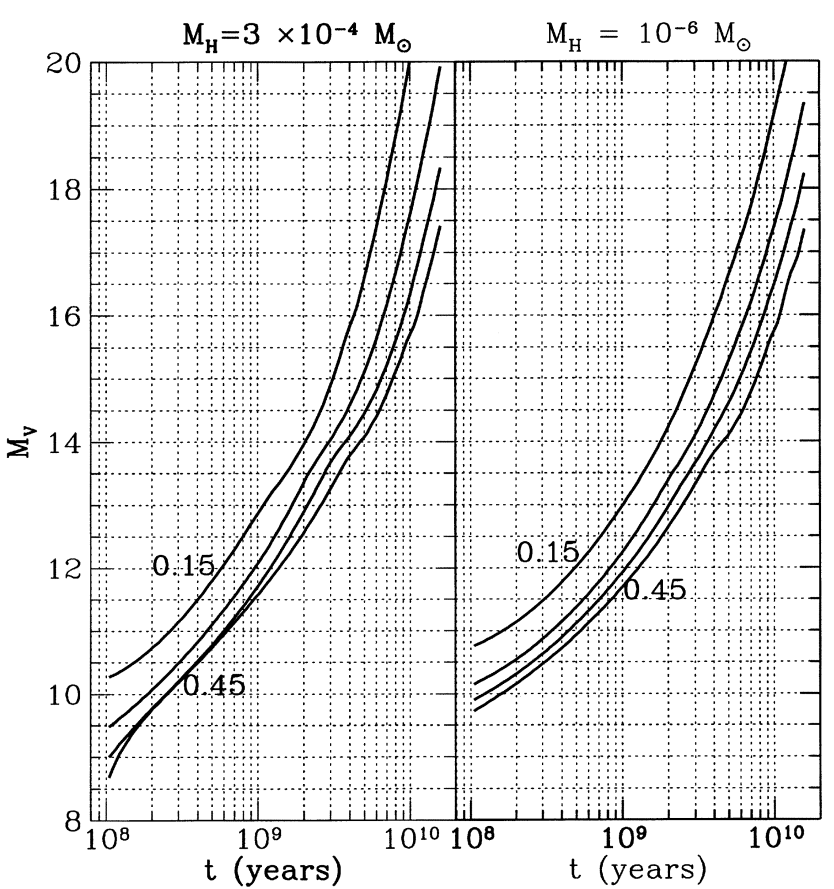

Figure 13. $V$-band cooling sequences. We show here the absolute $V$ magnitude determined from our cooling sequences. The curves are for $0.15,0.25,0.35$ and $0.45 \mathrm{M}_{\odot}$. 


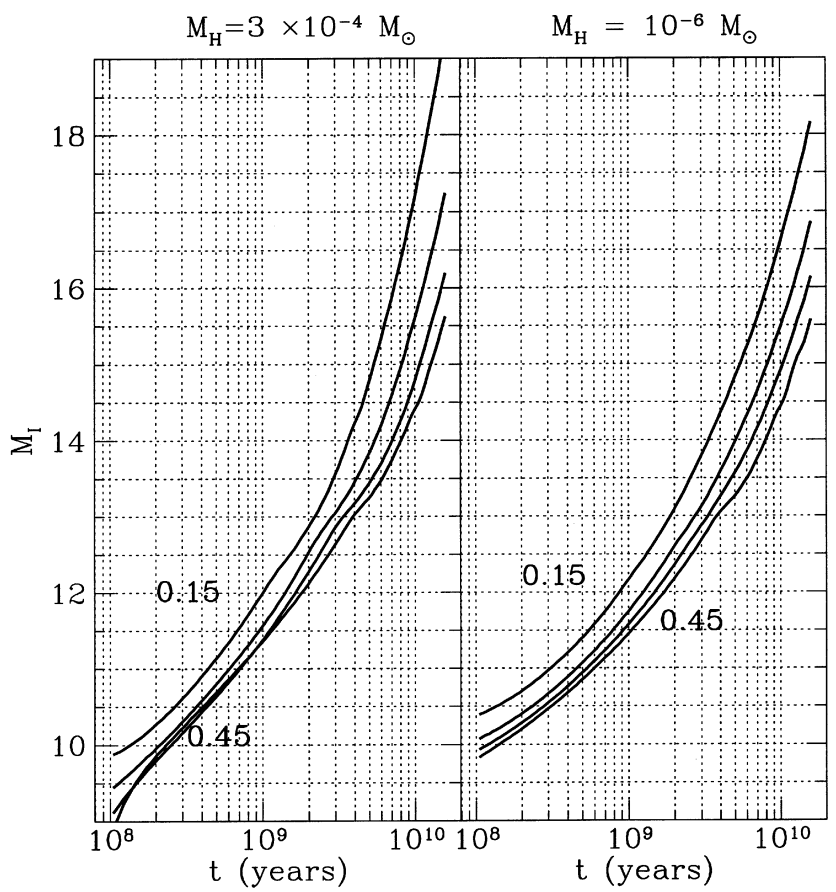

Figure 14. $I$-band cooling sequences. As for Fig. 13, but for absolute $I$ magnitude.

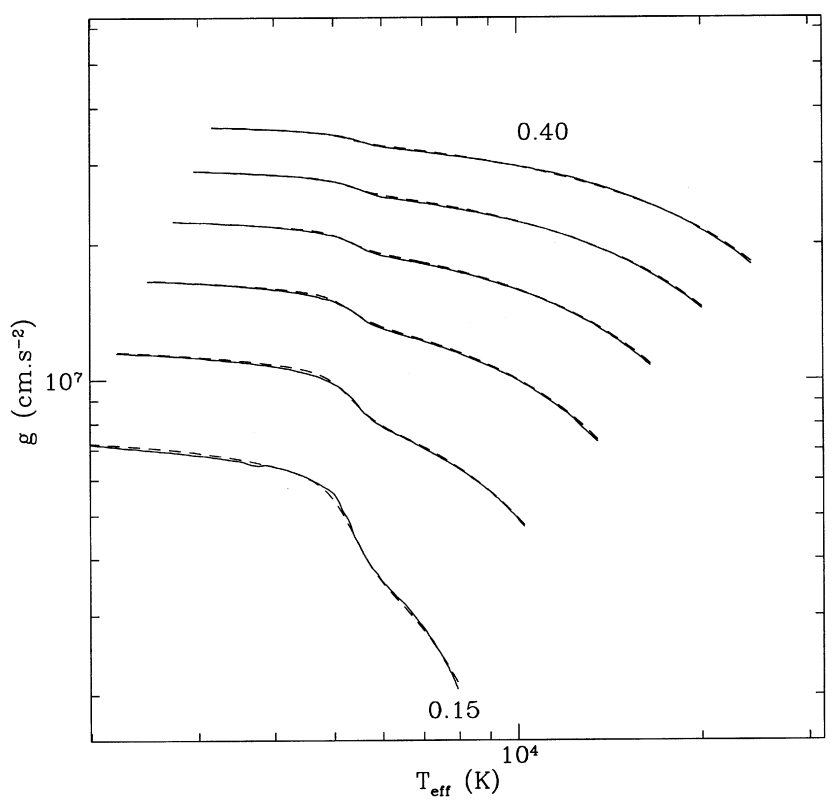

Figure 15. The gravity-effective temperature relation. The solid lines represent the true $g-T_{\text {eff }}$ curves, and the dashed lines are the fits given by equations (1)-(5).

for the thick hydrogen envelope, and

$$
\begin{aligned}
& a_{1}=3.79 \times 10^{-7}\left(\frac{M}{\mathrm{M}_{\odot}}\right)^{-2.18}, \\
& a_{2}=1.68 \times 10^{8}\left(\frac{M}{\mathrm{M}_{\odot}}\right)^{1.55}, \\
& a_{3}=650
\end{aligned}
$$

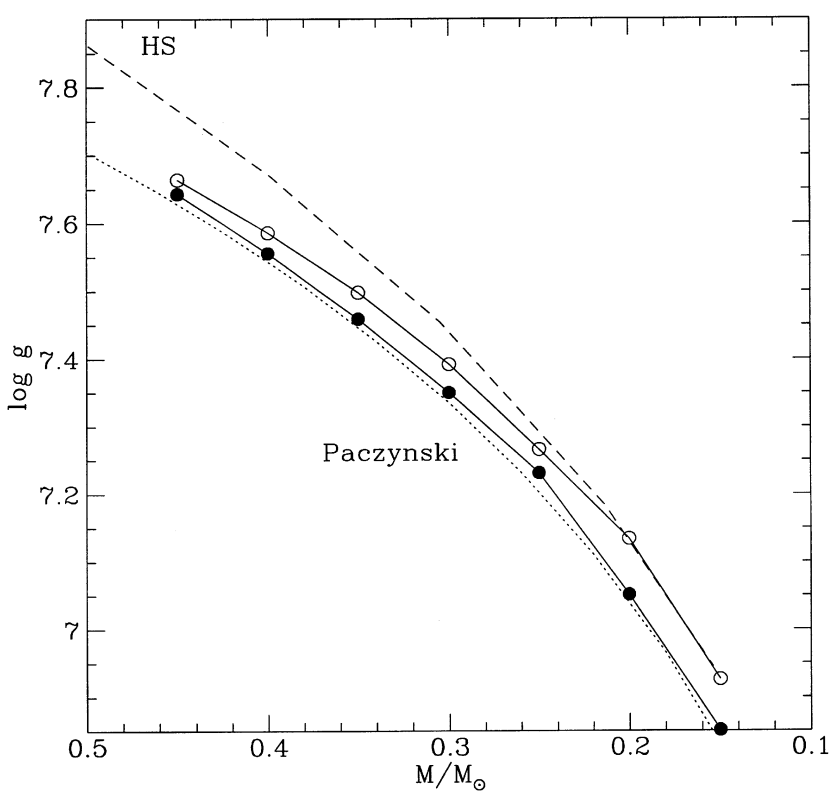

Figure 16. The $T=0$ mass-radius relation. The dotted line is the gravity as determined from the Paczynski (1967) mass-radius relation. The dashed line was obtained using the Hamada \& Salpeter (1961) pure helium massradius relation. The filled circles are for the thick $\mathrm{H}$ envelope models (equation 9), and the open circles for the thin $\mathrm{H}$ envelope models (equation 10).

for the thin hydrogen envelope and $M \leq 0.4 \mathrm{M}_{\odot}$. Fig. 15 compares the fits to the proper curves for the case of a thick envelope.

We may compare the $T_{\text {eff }}=0$ limit of (1) with various wellknown $T=0$ mass-radius relations. Converting the above into mass-radius relations and extrapolating to the $T=0$ limit we get

$\frac{R}{\mathrm{R}_{\odot}}=0.013\left(\frac{M}{\mathrm{M}_{\odot}}\right)^{-0.32}$ (thick $\left.\mathrm{H}\right)$,

$\frac{R}{\mathrm{R}_{\odot}}=0.013\left(\frac{M}{\mathrm{M}_{\odot}}\right)^{-0.28}$ (thin $\mathrm{H}$ ).

This is very close to the often-used mass-radius relationship of Paczynski (1967) for low-mass degenerate dwarfs, $R / \mathbf{R}_{\odot}=0.013\left(M / \mathrm{M}_{\odot}\right)^{-1 / 3}$. In Fig. 16 we compare our $T=0$ curves with the Paczynski relation and that of Hamada \& Salpeter (1961).

\section{CONCLUSION}

We have presented a set of cooling sequences for low-mass helium white dwarfs of different masses and with different masses of surface hydrogen. We provide blackbody absolute magnitudes and surface gravity-effective temperature relations as an aid to the analysis of future observations. In Paper II we shall apply these models to the optical observations of the companions to millisecond pulsars in order to derive cooling ages.

\section{ACKNOWLEDGMENTS}

The authors thank Marten van Kerkwijk and Yanqin Wu for lengthy discussions about white dwarf physics, and Glenn Soberman for helping with the initial conditions for our models. The generosity of D. Saumon, G. Fontaine, I. Mazzitelli, F. Rogers and C. Iglesias in providing their microphysical results is also appreciated. We also 
thank the referee, Dr D'Antona, for some insightful comments on the atmospheric physics.

\section{REFERENCES}

Alberts F., Savonije G. J., Pols O. R., van den Heuvel E. P. J., 1996, Nat, 380, 676

Bergeron P., Saumon D., Wesemael F., 1995, ApJ, 443, 764

Bergeron P., Wesemael F., Fontaine G., 1991, ApJ, 367, 253

Bergeron P., Wesemael F., Fontaine G., 1992, ApJ, 387, 288

Bessell M. S., 1979, PASP, 91, 589

Böhm K.-H., Carson T. R., Fontaine G., Van Horn H. M., 1977, ApJ, 217, 511

Borysow A., Frommhold L., 1990, ApJ, 348, L41

D’Antona F., Mazzitelli I., 1989, ApJ, 347, 934

D'Antona F., Mazzitelli I., 1990, ARA\&A, 28, 139

Dupuis J., Fontaine G., Pelletier C., Wesemael F., 1992, ApJS, 82, 505

Eggleton P. P., 1971, MNRAS, 151, 351

Fontaine G. Graboske H. C., Van Horn H. M., 1977, ApJS, 35, 293

Fowler W., Caughlan G. R., Zimmerman B. A., 1975, ARA\&A, 21, 165

Hamada T., Salpeter E. E., 1961, ApJ, 134, 683

Henyey L. G., Wilets L., Böhm K. H., LeLevier R., Levee R. D., 1959, ApJ, 129,628

Henyey L. G., Forbes J. E., Gould N. L., 1964, ApJ, 139, 306

Hubbard W. B., Lampe M., 1969, ApJS, 18, 297

Iben I., Tutukov A. V., 1986, ApJ, 311, 742

Itoh N., Kohyama Y., 1983, ApJ, 275, 858

Itoh N., Mitake S., Iyetomi H., Ichimaru S., 1983, ApJ, 273, 774

Itoh N., Matsumoto N., Seki M., Kohyama Y., 1984, ApJ, 279, 413

Kippenhahn R., Kohl K., Weigert A., 1967a, Z. Astrophys., 66, 58

Kippenhahn R., Weigert A., Hofmeister E., 1967b, in Alder B., Fernbach S., Rotenberg M., eds, Meth. Comput. Phys., Vol. 7. Academic Press, New York, p. 129

Koester D., Schönberner D., 1986, A\&A, 154, 125

Lenzuni P., Chernoff D. F., Salpeter E. E., 1991, ApJS, 76, 759 (LCS)

Marsh T. R., Dhillon V. S., Duck S. R., 1995, MNRAS, 275, 828

Mazzitelli I., 1989, ApJ, 340, 249

Mestel L., 1952, MNRAS, 112, 583

Mitake S., Ichimaru S., Itoh N., 1984, ApJ, 277, 375

Munakata H., Kohyama Y., Itoh N., 1985, ApJ, 296, 197

Paczynski B., 1967, Acta Astron., 17, 287

Phinney E. S., Kulkarni S. R., 1994, ARA\&A, 32, 591

Pols O., Tout C. A., Eggleton P. P., Han Z., 1995, MNRAS, 274, 964

Pylyser E. H. P., Savonije G. J., 1988, A\&A, 191, 57

Rogers F. J., Iglesias C. A., 1992, ApJS, 79, 507

Saumon D., Chabrier G., Van Horn H. M., 1995, ApJS, 99, 713

Schatzmann E., 1958, White Dwarfs. North-Holland, Amsterdam

Sweigart A. V., Gross P. G., 1978, ApJS, 36, 405

Winget D. E., Van Horn H. M., 1987, in Davis Philip A. G., Hayes D. S., Liebert J. W., eds, Proc. IAU Colloq. 95, The Second Conference on Faint Blue Stars. L. Davis Press, Schenectady, NY, p. 363

Wood M. A., 1992, ApJ, 386, 539

Zhang C., Borysow A., 1995, ApJ, 441, 960

\section{APPENDIX A: BRIEF NUMERICAL DETAILS}

It is our intention to make both the results and the cooling code publicly accessible. To obtain the most up-to-date version of the code, email hansen@cita.utoronto.ca. In this brief appendix we outline the version of the stellar structure equations that our program is designed to solve.

The four stellar structure equations are

$$
\begin{aligned}
\frac{\mathrm{d} P}{\mathrm{~d} r} & =-\rho \frac{G M(r)}{r^{2}}, \\
\frac{\mathrm{d} M}{\mathrm{~d} r} & =4 \pi r^{2} \rho,
\end{aligned}
$$

Table A1. Symbols used in the Appendix.

\begin{tabular}{lcc}
\hline Physical quantity & Symbol & Transformed \\
Pressure & $P$ & $\pi$ \\
Temperature & $T$ & $\theta$ \\
Luminosity & $L$ & $\ell$ \\
Radius & $r$ & $x$ \\
Mass & $m$ & $\xi$ \\
Time & $t$ & $\tau$ \\
Density & $\rho$ & $\phi$ \\
\hline
\end{tabular}

$\frac{\mathrm{d} L}{\mathrm{~d} r}=4 \pi r^{2} \rho \epsilon$

$\frac{\mathrm{d} T}{\mathrm{~d} P}=\frac{T}{P} \nabla$,

where $\nabla$ in equation (A4) is determined by

$\nabla=\frac{3}{16 \pi a c} \frac{\kappa}{T^{3}} \frac{L(r)}{G M(r)}$

for a radiative atmosphere or from a mixing length calculation for a convective atmosphere. The function $\epsilon$ contains both the nuclear energy generation terms and the thermal and gravitational loss terms, as well as the neutrino energy losses. We use the transformation of variables from (see Table A1 for definition of symbols)

$$
\begin{aligned}
x & =r / 10^{11} \mathrm{~cm}, \\
\ell & =L / L_{0}, \\
\pi & =\ln P / P_{0}, \\
\theta & =\ln T / T_{0}, \\
\phi & =\ln \rho / \rho_{0}, \\
\bar{\epsilon} & =\frac{L_{0}}{M_{0}} \epsilon, \\
\tau & =t \frac{L_{0} \rho_{0}}{M_{0} P_{0}}, \\
m & =M / M_{0}=1-\exp (-\xi),
\end{aligned}
$$

with the normalizations

$P_{0}=2.123 \times 10^{14} \mathrm{dyn} \mathrm{cm}^{-2}\left(\frac{M_{0}}{\mathrm{M}_{\odot}}\right)^{2}$,

$\rho_{0}=0.159 \mathrm{~g} \mathrm{~cm}^{-3} \frac{M_{0}}{\mathrm{M}_{\odot}}$,

$T_{0}=3.568 \times 10^{6} \mathrm{~K}\left(\frac{M_{0}}{\mathrm{M}_{\odot}} \frac{L_{0}}{\mathrm{~L}_{\odot}}\right)^{1 / 4}$

and

$L_{0}=100 \mathrm{~L}_{\odot}$.

The set of equations that we actually solve numerically is

$\frac{\mathrm{d} x}{\mathrm{~d} \xi}=\frac{\mathrm{e}^{\xi-\phi}}{x^{2}}$ 
$\frac{\mathrm{d} \ell}{\mathrm{d} \xi}=\mathrm{e}^{\xi}\left[\frac{Q}{\nabla_{\mathrm{ad}}} \mathrm{e}^{\pi-\phi}\left(\nabla_{\mathrm{ad}} \frac{\mathrm{d} \pi}{\mathrm{d} \tau}-\frac{\mathrm{d} \theta}{\mathrm{d} \tau}\right)-\bar{\epsilon}_{\nu}+\bar{\epsilon}_{\text {nuc }}\right]$,

$\frac{\mathrm{d} \pi}{\mathrm{d} \xi}=-\left(1-\mathrm{e}^{-\xi}\right) \frac{\mathrm{e}^{\xi-\pi}}{x^{4}}$

$$
\frac{\mathrm{d} \theta}{\mathrm{d} \xi}=-\left(1-\mathrm{e}^{-\xi}\right) \frac{\mathrm{e}^{\xi-\pi}}{x^{4}} \nabla
$$

[Q $\left.=-(\partial \ln \rho / \partial \ln T)_{P}, \quad \nabla_{\mathrm{ad}}=(\partial \ln T / \partial \ln P)_{S}\right]$. This system of equations is solved on a grid equally spaced in $\xi$. The external boundary condition is determined by integrating inwards using an adaptive stepsize algorithm starting with a grey atmosphere solution to determine $\pi$ and $\theta$ at the outer grid point as a function of $\ell$ and $x$. The fitting procedure used is taken from the triangle method of Kippenhahn et al. (1967b). 